
cultures

Les cahiers de l'Acedle

\title{
Le plurilinguisme en Alsace : les représentations sociales comme ressources ou outils de la description sociolinguistique
}

\section{Arlette Bothorel-Witz}

\section{(2) OpenEdition \\ 1 Journals}

Édition électronique

URL : http://journals.openedition.org/rdlc/6255

DOI : ERREUR PDO dans /localdata/www-bin/Core/Core/Db/Db.class.php L.34 : SQLSTATE[HY000]

[1040] Too many connections

ISSN : 1958-5772

Éditeur

ACEDLE

\section{Référence électronique}

Arlette Bothorel-Witz, « Le plurilinguisme en Alsace : les représentations sociales comme ressources ou outils de la description sociolinguistique », Recherches en didactique des langues et des cultures [En ligne], 5 | 2008, mis en ligne le, consulté le 05 novembre 2019. URL : http://journals.openedition.org/ rdlc/6255 ; DOI : ERREUR PDO dans /localdata/www-bin/Core/Core/Db/Db.class.php L.34: SQLSTATE[HYO00] [1040] Too many connections

\section{(c) (†) $\ominus$}

Recherches en didactique des langues et des cultures is licensed under a Creative Commons AttributionNonCommercial-NoDerivatives 4.0 International License 


\title{
Le plurilinguisme en Alsace : les représentations sociales comme ressources ou outils de la description sociolinguistique
}

\author{
Arlette Bothorel-Witz \\ Département de Dialectologie \& Gepe (EA 1339), université Marc Bloch, Strasbourg 2, \\ France
}

\section{Résumé}

Dans nos recherches sur le contact des langues et le plurilinguisme en Alsace, une place centrale a été donnée aux représentations sociales telles qui ont été intégrées par un groupe ou un individu (représentations stabilisées) mais aussi telles qu'elles peuvent se reconstruire ou se modifier dans l'interaction. Á partir d'exemples relevant de trois études de terrain et de différents espaces sociolinguistiques, il est proposé de s'interroger sur l'apport du modèle des représentations pour la description sociolinguistique et, partant, sur les références théoriques qui permettent de rendre compte de la relative stabilité des représentations instituées, d'une part, de leur dynamique et de leurs fluctuations, d'autre part.

\section{Mots-clés}

plurilinguisme, contacts de langues, dialectes alsaciens, représentations sociales, psychologie sociale, fonctionnements discursifs

\begin{abstract}
In our research on language contact and plurilingualism in Alsace a central place is given to social representations as shared by a group or society (stabilised representations) as well as to those re-constructed or modified during individual interactions. Taking examples from three field studies in different sociolinguistic spaces, we propose to examine the contribution of the model of social representations for sociolinguistic description and, therefore, the theoretical references that account for the relative stability of social representations on the one hand, and their dynamics and fluctuations, on the other.
\end{abstract}




\section{Keywords}

plurilingualism, contacts between languages, Alsatian dialects, social representations, social psychology, discourse mechanisms

\section{Introduction}

Bien que le terrain alsacien ait suscité l'intérêt de nombreux chercheurs français et étrangers, force est de constater qu'une description sociolinguistique "globale" fait défaut. C'est, en conséquence, par approches successives et complémentaires, que nos travaux ${ }^{1}$ tendent à rendre compte d'une situation de plurilinguisme marquée par les changements de formes, de fonctions et de valeurs des variétés en présence, par l'évolution des pratiques et par la restructuration des répertoires des locuteurs. Dans le cadre d'un projet d'ensemble qui vise à comprendre la situation dans sa globalité contextuelle, nous avons cherché, en partant d'études préexistantes et de nouvelles recherches empiriques, à cerner les divers paramètres en jeu, (historiques, politiques, économiques...) à diversifier les terrains d'observation et les acteurs, à multiplier les outils descriptifs et les références épistémologiques. Le modèle des représentations - qui tend à rendre compte de la perception collective et individuelle que les locuteurs ont de leurs pratiques linguistiques et de celles des autres - constitue l'une des options épistémologiques retenues pour appréhender "le fonctionnement complexe d'un ensemble d'éléments" (Calvet, 1999 : 15) constitutif du plurilinguisme ${ }^{2}$ en Alsace. Nous nous proposons, après avoir précisé les cadres théoriques de référence, de montrer de quelle manière les représentations sociales (R.S), objectivées dans le discours épilinguistique ${ }^{3}$, constituent un outil ou une ressource pour une description sociolinguistique et comment elles contribuent à rendre compte de la complexité du plurilinguisme en Alsace.

\footnotetext{
${ }^{1}$ Voir, à ce sujet, Huck, Bothorel-Witz, Geiger-Jaillet, 2007 : 90-100.

${ }^{2}$ Le terme "plurilinguisme" renvoie ici à une situation de contacts de variétés qui sont présentes dans la communication sociale et dans le répertoire linguistique des locuteurs (Juillard, $2007: 235$ ).

${ }^{3}$ Le terme épilinguistique est employé dans le sens de Canut (2007: 50), de sorte qu'il renvoie à "des discours autonomes sur des lectes et des formes et activités langagières" (les siens et ceux des autres).
} 


\section{Les terrains, le cadre méthodologique et théorique}

\subsection{Terrains et espaces sociolinguistiques ${ }^{4}$}

En abordant la situation alsacienne sous l'angle des contacts de langues et d'un plurilinguisme complexe, une place centrale a été donnée aux RS dans trois études de terrain. La première de ces recherches empiriques, commencée à la fin des années 80 (cf. annexe 1, projet sur "la conscience linguistique des locuteurs dialectophones alsaciens"), vise à comprendre comment les locuteurs alsaciens (aux profils et aux habitus divers) (re)construisent et se représentent leur univers sociolinguistique et, partant, le plurilinguisme alsacien.

En retenant la même démarche et en gardant les mêmes références épistémologiques et méthodologiques, nos investigations ont été étendues récemment à deux autres terrains d'enquête et d'observation : l'institution scolaire (cf. annexe 2, programme Interreg III C) et les entreprises à vocation internationales implantées en Alsace (cf. annexe 3, projet intégré Dylan). Alors que les premières recherches (menées dans le cadre du projet "Conscience linguistique des locuteurs dialectophones alsaciens") constituent en quelque sorte notre base de référence, l'élargissement à d'autres "espaces sociolinguistiques" et à d'autres acteurs sociaux vise à mieux saisir le plurilinguisme alsacien dans sa globalité contextuelle. Tout en présentant des particularités de fonctionnement, ces espaces (école, entreprises) ne peuvent être détachés de l'environnement sociolinguistique alsacien dans lequel ils s'insèrent : ils révèlent les identités enchâssées des acteurs et font apparaître de nouvelles polarités autour desquelles se structurent ou se restructurent les rapports de force entre les variétés en présence. Sous l'influence de variétés exogènes (l'anglais en particulier), les variétés endogènes (dialectes, variétés de français, allemand endogène), se voient dotées de nouvelles fonctionnalités ou valeurs. Il s'ensuit que les résultats issus de la comparaison de ces trois études de terrain viennent nuancer, compléter, voire rectifier la description initiale en même temps qu'ils permettent "d'avancer vers la formulation d'une synthèse interprétative relativement globale" (Blanchet, 2000 : 55).

\footnotetext{
${ }^{4}$ Pour la notion d'espace sociolinguistique, voir Juillard (2007 : 237) :"Des lieux, des acteurs sociaux, des types d'activités, notamment discursives, et des thématiques de discours définissent des espaces sociolinguistiques au sein desquels la parole plurilingue se déploie en situation".
} 


\subsection{Le recueil des données discursives}

Puisque le discours est, par excellence, le lieu d'existence des RS, nous avons opté pour la méthodologie de l'entretien thématique qui donne aux personnes interrogées la possibilité de développer leur position, d'éluder le sujet, voire de faire des digressions. Malgré l'adaptation du guide thématique aux spécificités de chacun des trois terrains, un certain nombre de questions communes sur les fonctions des variétés associées aux pratiques, sur les représentations des normes, sur celles des variations, du bi-/plurilinguisme, sur l'autoévaluation des compétences, etc. ont permis de mettre en contraste les faits représentationnels ressortant des différentes études de terrain.

\subsection{Le repérage des $\mathrm{RS}$ dans le discours et leur traitement}

La focalisation sur les RS en tant que ressources pour la description sociolinguistique nous oblige à préciser comment les RS ont d'abord été repérées, puis traitées dans le discours. Dans le mesure où le "discours sur" ne peut être posé comme l'équivalent des "représentations", il se pose inévitablement la question de la sélection des fragments de discours dans lesquels les RS s'actualisent. Or, le choix de ces observables est déterminé par le cadre théorique (linguistique ou psychosociologique) qui sous-tend l'analyse des RS.

Dans la phase de repérage des représentations dans le discours, nous privilégions la conception psychosociologique des RS qui met l'accent sur le caractère partagé d'un savoir diffusé dans un lieu ou dans un groupe social. En transférant ainsi la notion de RS issue de la psychologie sociale dans notre propre champ, nous en adoptons aussi les traits de définition les plus saillants.

- Les RS - qui sont des éléments de la conscience sociale extérieure aux individus sont définies comme "un ensemble organisé de cognitions relatives à un objet" (Flament, $1994: 37$ ) ;

- la représentation est représentation d'un objet (langues / pratiques, compétences, bilinguisme, etc.). Se représenter est, en conséquence, une activité mentale par laquelle le sujet se rapporte à un objet et traduit sa relation symbolique à l'objet ;

- les RS constituent des "systèmes d'interprétation régissant notre relation au monde et aux autres, [qui] orientent et organisent nos conduites et les communications sociales" (Jodelet, 1989 : 36). Elles suscitent une prise 
de position et "ont pour fonction de fournir [...] des interprétations utiles à une activité en cours [...]" (Py, $2004: 8)$;

- Les RS se construisent dans le cadre d'un processus d'échange et d'interaction.

Concernant le repérage des RS, nous admettons, en conséquence, que c'est la thématisation à laquelle donne lieu un objet social dans l'échange qui est susceptible de le transformer en objet de représentation et de discours (Gajo, $2003: 527)^{5}$. Les RS qui sont, implicitement ou explicitement, activées par la thématisation s'expriment alors à travers un faisceau de connaissances, d'opinions, de croyances, d'attitudes qui sont largement partagées et qui "définissent un espace de signification commun à tous les membres du groupe" (Moliner, Guterman, 2004: 2.2.). En adoptant ce point de vue (que justifie l'orientation sociolinguistique de notre recherche), l'accent est mis, dans une première phase de traitement des RS dans le discours, d'une part, sur l'aspect déclaratif des représentations (ce qui est dit) et, d'autre part, sur des contenus ${ }^{6}$ qui font l'objet d'un très large consensus. On met ainsi en relief ce que les tenants de la théorie du noyau (Abric, 1994 ; Flament, 1989 ; Guimelli, 1994) considèrent comme les éléments centraux de la représentation et comme "une infrastructure relativement stable, anonyme et indépendante des contextes dans lesquels la représentation est mobilisée" (Py, 2000 : 118). Il s'ensuit que ces RS constituent un réseau de "disponibilités sociales", extérieures à l'interaction (Gajo, 2003 : 526), "[qui] ne requièrent aucun travail autre que l'acte de leur mise en œuvre énonciative" (Py, 2004 : 8).

Si les RS sont objectivées dans le discours, elles sont parallèlement configurées par et pour le discours (Gajo, 2003: 520), ce qui signifie que le discours fournit aux énonciateurs particuliers les ressources nécessaires pour reconstruire, voire reconfigurer ou modifier, les éléments représentationnels partagés. En fonction de leurs expériences personnelles et/ou des contraintes contextuelles, les énonciateurs peuvent (ou non) prendre à leur compte les représentations instituées, exprimer leur appartenance à un groupe ou construire leur identité

\footnotetext{
${ }^{5}$ Malgré ces précautions théoriques et méthodologiques, on ne peut cependant prétendre à une exploitation exhaustive des RS. D'une part, toutes les RS ne sont pas visibles ou lisibles dans le discours. D'autre part, comme le rappelle Gajo "tout dans le discours ne donne certainement pas lieu de manière égale à thématisation et encore moins à représentation" (Gajo, 2003: 527). Même si l'entretien thématique peut conduire à l'activation d'une représentation chez l'informateur, il n'en reste pas moins vrai que certains objets ne sont pas transformés en objet de représentation et, en conséquence, de discours. L'objet peut ne pas être présent dans le réseau commun de disponibilités sociales.

${ }^{6}$ Comme le rappellent Moliner et Guterman (Moliner, Guterman, 2004 : 2.4.), le contenu des RS comporte des éléments de nature diverse (définitoires, descriptifs, explicatifs, évaluatifs, ...) qui jouent des rôles différents.
} 
propre. Ces modulations individualisées constituent les éléments ou les "schèmes périphériques" au noyau central. En assurant "les applications du noyau à des objets et à des contextes déterminés, par des personnes particulières" (Py, 2000: 118), ces éléments périphériques révèlent en même temps le caractère instable et variable du traitement des $\mathrm{RS}$ par les énonciateurs.

Pour rendre compte de ces différentes "épaisseurs" discursives des RS, nous optons pour une stratégie de triangulation qui conduit à combiner les deux conceptions (psychosociologique et linguistique) des RS et à les aborder dans leurs doubles dimensions, homogène et hétérogène, collective et individuelle. Comme le rappellent très justement Billiez et Millet, dans la mesure où le discours individuel est traversé par tous les autres, "le Même et l'Autre s'enlacent" (Billiez \& Millet, 2000 : 39), de sorte que négliger l'une des deux composantes revient à perdre une vision globale des phénomènes représentationnels et à effacer les traces de la variation. Ce n'est qu'en répondant à cette double exigence théorique que le modèle des RS peut contribuer à mieux cerner la complexité d'une situation plurilingue et les rapports de force variables entre les variétés présentes dans la communication sociale et dans le répertoire linguistique des locuteurs.

\begin{tabular}{|ll}
\hline Conception psychosociologique des RS & Conception linguistique des RS \\
RS actualisées dans le discours & RS configurées par et pour le discours \\
Disponibles, prêtes à l'emploi & Reconstruites par un énonciateur \\
Partagées, plus stables & Plus instables, plus variables \\
Traitement du contenu &
\end{tabular}

Figure 1 - Deux conceptions des représentations sociales

\section{Les RS comme ressources de la description sociolinguistique}

Pour des raisons heuristiques, nous évaluerons successivement les apports d'une analyse que sous-tend, d'une part, la conception psychologique des RS, d'autre part, la conception linguistique. 


\subsection{Les RS comme pré-constructions ou disponibilités sociales actualisées dans le discours}

Le point de vue psychosociologique adopté conduit, dans une phase initiale, à identifier les "disponibilités sociales" matérialisées dans le discours.

\subsubsection{Les RS partagées (indépendamment du terrain)}

Les trois études retenues (cf. supra) font ressortir des contenus représentationnels partagés qui sont indépendants des terrains et des acteurs retenus dans nos trois études. Cette forme de stabilité se manifeste, pour partie, dans les représentations des dialectes et de l'allemand.

Les représentations des variétés dialectales sont, pour la majorité des locuteurs alsaciens, sur-déterminées par la vision idéalisée du français. Lorsqu'ils sont amenés à se prononcer sur ce que serait un dialecte, ils le font très naturellement par opposition à leur représentation de ce qu'est une "langue" (en l'occurrence le français). Comme dans d'autres situations de plurilinguisme, le caractère éminemment oral du dialecte renvoie à son statut de non-langue et, partant, à l'absence d'écriture, de codification et de grammaire. Le fait que les variétés dialectales obéissent aussi à une syntaxe, à une morphologie et à une phonétique ne fait pas partie des disponibilités sociales prêtes à l'emploi.

La vision idéalisée d'un dialecte "pur", "authentique", non marqué par les interférences avec le français, est très largement prédominante, quel que soit le profil des acteurs : ce qui fait figure de "norme dialectale" est identifié à la variété sociolectale du groupe de locuteurs âgés, ruraux, peu mobiles qui constitue l'objet mythique de la dialectologie traditionnelle. Lorsque l'alsacien est envisagé sous l'angle de sa fonctionnalité dans les pratiques quotidiennes, l'accent est surtout mis sur son utilité dans les interactions avec les personnes âgées (cf. séquence 1 et 2 ) dont on suppute qu'elles pourraient ne pas bien parler / comprendre le français.

\section{Séquence 1 : Jeune femme de 22 ans, Professeure des écoles}

$$
\begin{aligned}
& E \text { : on dit parfois * que l'usage du dialecte est lié à une situation * à un } \\
& \text { interlocuteur * qu'en pensez-vous/ } \\
& \mathrm{I}: \text { euh euh * oui euh * j'pense que euh * j'parlerais pas en dialecte avec euh * } \\
& \text { avec * avec une personne euh * de ma génération * j’pense que j'utiliserais } \\
& \text { automatiquement le français et euh * peut-être qu'avec une euh * une personne }
\end{aligned}
$$


plus âgée $(. .$.$) * comme je connais le dialecte * j’pense que je parlerais *$ probablement $(\ldots) *$ le dialectel

\section{Séquence 2: Chef de région des vendeurs dans une filiale française d'un groupe allemand (Bas-Rhin)}

KZ : d'accordl et au travail * vous parlez quelles langues/

WUB : ben ça dépend ici au niveau du siège on parle français * à moins qu'on convoque des vendeurs alsaciens- - enfin des vendeurs de l'ancienne génération on va dire * qui parlent un peu l'alsacien auquel cas on parle * on est amené à parler en alsacienl

Le discours sur l'allemand révèle d'autres éléments largement partagés. C'est ainsi que son importance est régulièrement majorée en raison de la proximité de l'Allemagne, de l'histoire de l'Alsace et d'une forme de proximité interlinguistique avec l'alsacien. En d'autres termes, la place qui revient à l'allemand, dans les représentations, est essentiellement associée à un espace régional, voire transfrontalier. Le rôle qu'il pourrait, en revanche, jouer à l'international n'est pas, quel que soit le terrain, un objet de représentation et de discours. Parallèlement, l'allemand est relié à la tradition et à un passé plus ou moins révolu. Ce positionnement face à l'allemand, qui est très présent chez les apprenants pour qui l'anglais est le vecteur de la modernité (cf. annexe 2), prend, comme le montre la séquence qui suit, tout son sens dans les entreprises :

\section{Séquence 3: Responsable du service achat dans une filiale française d'un groupe allemand}

KZ : vous parliez de la place de l'anglais * est ce que la situation a évolué de ce point de vue/ votre maison mère est pourtant allemande/

WUE : ben oui * forcément puisQUE * quand je remonte à * l'année 89 * l'année où je suis rentré ici * à l'époque on achetait $70 \%$ des produits chez W. Allemagne * donc on pouvait se contenter très largement de ne parler que l'alleMAND * et ça suffisait * aujourd'hui avec l'internationnalisation * il est clair que l'anglais est presQU UN impéRAtif * enFIN c'est un impéraTIFI

Les facteurs qui conduisent à ce rapport de force entre l'allemand et l'anglais dans les entreprises sont régulièrement associés à la mondialisation, à de nouveaux marchés (en Asie en particulier), à la domination économique des Ėtats-Unis, à la compétitivité et au souci d'anticipation et de développement des entreprises. Comme le dit l'un des acteurs : "c'est l'envergure de l'entreprise qui donne l'importance de la langue" (celle de l'anglais !). 
Finalement, le discours sur les compétences linguistiques révèle des visions partagées qui sont déterminées par l'idéal-langue de référence et la crainte de la faute. Chez les locuteurs alsaciens, l'auto-évaluation des compétences en français (qui révèle régulièrement une insécurité linguistique plus ou moins prononcée selon les acteurs) se fait par rapport au codenorme prescriptif de l'écrit et à "un faisceau de recommandations en matière de bien dire (et de bien écrire, [...] dont la valeur de référence est inculquée, puis entretenue, par diverses autorités institutionnelles ou non" (Singy, 1996 : 25). Ce souci de conformité à une norme et le sentiment de ne pas pouvoir réellement s'y conformer transparaît dans la plupart des discours sur le français :

\title{
Séquence 4 : PDG d'une filiale française d'un groupe américain
}

\author{
WRF : écouTEZ si JE * si je lis une LEttre euh et que y a des des FAUtes de \\ synTAxe ou qu'i y a des FAUtes d'orthoGRAphe euh: et puis ça aRRIve tous \\ les jours J'Al euh: une présompTION un: peu négativel \\ $A B$ : mais là vous parlez de ce qui est écrit * est-ce que ce serait pareil lorsque \\ vous parlez justement/ \\ WRF : je pense qu'il faudrait pouvoir parler euh sans fautes et qu'on gagne \\ beaucoup à parler sans fautes * ce qui est pas mon cas d'ailleursl
}

Ce sont ces mêmes rapports à la normativité qui conditionnent les représentations du plurilinguisme comme autant de monolinguismes juxtaposés se traduisant par une double compétence équilingue dans ce que les locuteurs considèrent comme des "vraies langues".

\subsubsection{Les fluctuations que révèlent les pré-constructions actualisées dans le discours}

Marqués qu'elles sont par la conception sociopsychologique des RS, certaines descriptions macro-sociolinguistiques se limitent à mettre en relief, comme nous venons de le faire, la dimension sociale et partagée des représentations. Ce faisant, elles négligent une forme de variabilité ou de fluctuations interdiscursives que révèle une analyse plus fine du discours épilinguistique. Afin de rendre compte des possibles apports d'une telle analyse, nous avons retenu deux formes de variabilité des RS actualisées dans le discours. La première relève du jeu de l'interdiscursivité ${ }^{7}$, la deuxième d'une dialectique de minoration / majoration des objets

\footnotetext{
${ }^{7}$ Voir aussi Canut, 2000.
} 
de représentation. Une autre forme de variabilité des RS est, finalement, reliée au choix que nous avons fait de diversifier les terrains et les espaces sociolinguistiques.

\section{Les fluctuations interdiscursives}

Dans la mesure où les RS sont organisées en systèmes socio-cognitifs, elles constituent un tout indissociable. De même qu'on ne peut détacher un objet de représentation et de discours des autres objets, on ne peut isoler, les uns des autres, les éléments et contenus représentationnels se rapportant à un même objet. Cette conception structurale des RS (issue de la psychologie sociale) amène à mettre en regard des parts éparses du discours qui font ressortir des fluctuations interdiscursives souvent considérées à tort comme des "contradictions" internes (Canut, 2000: 93). Pris dans l'interdiscursivité, les acteurs construisent des représentations dont les variations sont, entre autres, marquées par le point de vue adopté (identitaire, idéologique, politique, économique...). Ces positionnements, apparemment paradoxaux, se manifestent, en particulier, dans les représentations des liens d'apparentement entre l'alsacien et l'allemand. Dans les analyses de "la conscience linguistique des locuteurs alsaciens", la question (à forte charge idéologique) de la catégorisation de l'alsacien comme dialectes de l'allemand révèle, pour l'essentiel, deux types de stratégies. La plus répandue conduit les acteurs à "autonomiser" les variétés dialectales en les décrochant, indépendamment de l'empirie, de l'allemand et en enfermant l'Alsace dans un espace clos. Cette individuation se manifeste aussi dans le glottonyme "alsacien". Pour échapper à la catégorisation de l'alsacien comme un dialecte de l'allemand et, parallèlement, à une redéfinition des frontières subjectives, une autre stratégie consiste à rechercher un compromis. En optant pour une catégorisation ontologique en quelque sorte, le dialecte est identifié à un "entre-deux langues", un "mélange", pourtant dévalorisé dans les commentaires métalinguistiques en ce qu'il viole une norme. Le métissage revêt ici une valeur symbolique : il renvoie à une situation d'exception qui s'inscrit entre un intérieur (qu'il convient de définir) et un extérieur. Le principe dialogique - qui sous-tend cette stratégie - permet de dépasser les disparités culturelles et linguistiques. Dans les deux cas, le discours révèle une difficulté à s'inscrire dans une continuité historique.

Face aux enjeux éducatifs et économiques, l'accent est inversement mis sur la proximité de l'alsacien et de l'allemand. C'est ainsi que, pour les collégiens de la filière bilangue, l'alsacien, pourtant considéré comme une non-langue (par opposition au français) ou de 1'"allemand déformé", constitue un levier d'accès à l'apprentissage de l'allemand. Le dialecte, 
ainsi refonctionnalisé, acquiert un statut de médiateur, de passeur vers l'allemand, voire vers l'anglais (Castellotti, Moore, 2002 : 18, citant Coste, 2001).

Dans les entreprises, la proximité revendiquée entre les deux codes confère à la plupart des acteurs une compétence "naturelle" en allemand et une sécurité linguistique qui vient compenser l'insécurité que génère la prédominance redoutée de l'anglais. Dans le même ordre d'idées, la culture germanique des Alsaciens (dans sa double composante alsacienne et allemande) apparaît dans les RS, tout comme dans les brochures de promotion économique de l'Alsace, comme un atout aux yeux des investisseurs étrangers (allemands en particulier).

\section{Le jeu dialectique des processus de valuation}

Une analyse plus approfondie du contenu des représentations partagées, la mise en regard de parts éparses du discours fait ressortir une autre forme de variabilité. Elle se traduit par un jeu dialectique de processus de majoration et de minoration: un objet de représentation est minoré / majoré par rapport à un autre, un même objet est tantôt majoré, tantôt minoré, de sorte que les deux processus ne peuvent être dissociés l'un de l'autre. "C'est dans le rapport de tension entre major- et minor-, équilibre dynamique, que se joue le processus sans renouvelé de valuation" (Blanchet, 2005 : 34). Pour illustrer cette dialectique qui se manifeste sur tous les terrains retenus, nous avons retenu les processus antagonistes de majoration / minoration que révèlent les représentations des normes du français et de l'allemand, dans leurs composantes endogènes et exogènes.

Dans le discours, le "bon français" est identifié à un code-norme prescriptif et invariant qui implique l'idée de conformité à une règle. La survalorisation de ce modèle prescriptif a pour conséquence qu'on recherche des références normatives qui se situent toujours "ailleurs" et, de préférence, en dehors de l'espace alsacien. Selon les acteurs, il s'agit d'un ailleurs géographique (généralement le centre) et / ou d'un ailleurs social ou sociétal : dans la mesure où l'école représente l'espace institutionnel où s'exerce cette norme, les sujets qui ont la possibilité de s'approprier cette "langue légitime" constituent alors le groupe de référence. L'"ailleurs" est plus rarement de nature temporelle : les locuteurs alsaciens âgés ayant, pour partie au moins, été scolarisés en allemand pensent que le "bon" français est parlé par les générations qui ont appris le français à l'école. Il ressort de l'analyse des représentations des normes que l'idéal- langue existe en dehors des utilisateurs (alsaciens) et de son usage en contexte. Le représentant de cette norme fantasmée cumule plusieurs attributs : il est Français de 1'"intérieur", jeune, urbain et cultivé. La sujétion ou la soumission à ce modèle idéal, qui 
est hors de la portée des acteurs (il s'agit là constat qu'ils acceptent avec résignation), induit la minoration du français endogène. Sous la pression du modèle de prestige extérieur, les formes d'existence que prend, dans les usages, le français parlé en Alsace ne sont pas considérées comme des créations originales, mais comme un ensemble de déviances que matérialise "l'accent alsacien". Face au regard extérieur (francophone), supposé ou réel, la variété de français régional, qui constitue pourtant la forme la plus standardisée du répertoire linguistique d'un certain nombre de locuteurs alsaciens, est perçue, à l'oral, comme un mode d'expression indexant qui ne bénéficie pas de la même légitimité sociale que le français général. Il renvoie, semble-t-il, à la germanité, à l'inverse du dialecte qui reste emblématique de l'alsacianité. La dépréciation des marqueurs régionaux n'est toutefois pas systématique. Entre pairs linguistiques, l'"accent" - que tout le monde pense plus ou moins partagé constitue le signe d'appartenance à la même communauté. Il s'agit là d'une stratégie de compensation de la part de locuteurs en état de subordination linguistique et d'un véritable "acte d'identité" (Le Page, Tabouret-Keller, 1985).

Les représentations de ce que serait le "bon allemand", où il serait parlé et par qui, révèlent la même dynamique dialectique. En reproduisant le modèle français, on s'accorde à ne pas situer le "vrai" ou le "bon allemand" dans les régions frontalières, éloignées du centre. L'allemand endogène (dont l'usage dans les éditions bilingues de la presse régionale ou dans la littérature dite régionale ne devient pas objet de représentation) est assimilé à une variété qui est dotée des mêmes attributs négatifs que le français parlé en Alsace. En renvoyant aux seules productions orales des locuteurs dialectophones, l'allemand endogène est perçu comme un "mélange", qui est détérioré par les interférences avec le dialecte, de sorte qu'il se distingue très nettement de l'allemand "authentique", exogène.

Dans les deux cas, les représentations des normes du français et de l'allemand sont minorées lorsqu'elles se rapportent aux usages des Alsaciens; elles sont, inversement, majorées lorsqu'elles renvoient à des modèles exogènes auxquels les locuteurs ont le sentiment de ne pas pouvoir se conformer.

\section{Les variabilités induites par les terrains et les identités des acteurs}

La comparaison des trois études permet de montrer que la spécificité du terrain et celle des identités qu'y révèlent les acteurs peuvent, au-delà des aspects qui rencontrent un large consensus, révéler des différences entre les éléments représentationnels se rapportant à un même objet. Nous retenons pour illustration l'exemple du français et de l'alsacien. 
Alors que la valeur sociale du français, son statut de langue légitime, de vecteur de la modernité et sa polyfonctionnalité ressortent de toutes les études sur "la conscience linguistique des locuteurs alsaciens", les discours dans les entreprises mettent à jour de nouvelles valeurs et fonctions symboliques du français. Sous la menace que représente la toute-puissance réelle ou imaginaire de l'anglais, le français fonctionne comme un symbole identitaire dans des entreprises qui tiennent avant tout à préserver leur identité française. Cette fonction symbolique du français devient particulièrement manifeste dans les filiales de groupes étrangers, comme le montre l'extrait qui suit :

\section{Séquence 6 : PDG d'une filiale française d'un groupe américain}

$A B$ : vous dites le français est encore beaucoup utilisé icil

WRF : et bien euh: SI * si: on est en France * je pense: que: la: la première langue l'iMAGE qu'on doit doNNER c'est * c'est * c'est de parler le françaisl je crois que à terme les les multinationales * ça encore une fois ça ne regarde que moi * les multinationales vont réussir leur implantation dans la mesure où elles RESpectent euh: euh: la: * la: la spéci-la spécificité de l'endroit où elles s'implantentl si elles veulent * si elles veulent * si elles veulent LAminer les GENS ça VA pas marCHER à terme * à terme * ça fonctionne pası

Tandis que dans l'étude sur "la conscience linguistique des locuteurs alsaciens", l'analyse des discours fait ressortir une perte de fonctionnalité des dialectes (dans la vie sociale) qui est acceptée comme une donnée de fait et qui, partant, justifie le recours de plus en plus fréquent au français, les recherches portant sur l'école et, plus particulièrement, sur les entreprises viennent sensiblement corriger ce constat. Le dialecte est, non seulement, plus ou moins présent dans toutes les entreprises (dans les conversations informelles entre gens du cru, entre collaborateurs plus âgés, comme langue de connivence, etc.), mais il est re-fonctionnalisé dans les paroles de travail. C'est ainsi que dans la filiale française d'un groupe allemand et, plus précisément dans le secteur des ventes, le dialecte peut servir d'outil de communication dans les transactions avec des artisans, des petits patrons de l'industrie, dans le souci de se conformer à leur attente (réelle ou supposée) ou dans le sens d'un intérêt économique bien compris :

\section{Séquence 7: Chef de région des vendeurs dans une filiale française d'un groupe allemand}

WUB : moi j'ai le 88 le 57 le 67 et le 54 et donc quand je suis sur le 67 j'essaie de parler alsacien parce qu'on a remarqué que commercialement parlant 
certains certains artisans se libèrent sont plus plus libres * ils se sentent moins

** ils ont la barrière de la langue parce qu'ils ont peut-être du mal à s'exprimer en français donc nous on parlera alsacien * on arrive mieux et puis ça décante un peu la relation * quoil

L'alsacien peut aussi être présent dans les pratiques de la hiérarchie, ce qui témoigne sans doute de sa part d'une forme de stratégie linguistique. Dans une filiale française d'un groupe allemand, le directeur et les dirigeants parlent l'alsacien : "certains dirigeants parlent alsacien donc on peut entamer des discussions en alsacien". Le PDG de la filiale du groupe américain utilise volontiers le dialecte dans les ateliers de production pour marquer sa proximité avec les ouvriers :

\section{Séquence 8 : PDG d'une filiale française d’un groupe américain}

WRF : j'ai beauCOUP de contacts avec: euh: les les ouvriERS enfin les GENS qui travaillent aux chaînesl et LÀ si y a quelqu'un euh qui parle l'alsaCIEN euh je je leur parle en alsacienl

$A B$ : vous avez des raisons d`le faire/

WRF : OUI euh euh: oui peut-être parce que d'abord j'aime bien parLER I'alsaCIEN et ensuite pour un peu leur dire que euh: que euh: je suis avec eux:I je les connais bienl

Il ressort, finalement, des différentes formes de variations (limitées à quelques illustrations) que l'analyse des RS ne peut se limiter à la seule recherche des éléments stabilisés et des significations essentielles que les acteurs assignent collectivement aux objets de leur environnement social et sociolinguistique. Même si la psychologie sociale et, à sa suite, la sociolinguistique tendent à mettre l'accent sur le répertoire de représentations partagées, on ne peut pas, sous peine de réduire considérablement la richesse du contenu représentationnel, négliger la variabilité des RS et les facteurs qui la structurent. Les RS se situent au carrefour de l'homogène et de l'hétérogène et c'est dans les fluctuations entre ces deux pôles qu'elles révèlent toutes leurs ressources pour la description sociolinguistique. Bien que la conception psychosociologique des RS n'exclut pas, comme nous venons de le voir, la prise en compte d'une forme de variation, elle ne permet pas de rendre compte des liens entre les éléments centraux et périphériques des RS (Moliner, Martos, 2005) et de la diversité des traitements des RS par des énonciateurs. 


\title{
3.2. Les $R S$ configurées par et pour le discours
}

En cherchant à articuler la dimension collective et individuelle des RS, le Même et l'Autre, on est amené à opter pour une approche linguistique des RS qui vise à mettre à jour "la dimension polyphonique des représentations attribuées à des énonciateurs particuliers" (Mondada, 2004 : 258) et la dynamique des RS.

\subsubsection{Le choix des observables}

Il est déterminé par les objectifs de l'analyse des représentations configurées par et pour le discours. Bien que la grille de lecture retenue ne soit pas exclusive de toute autre, elle est, à ce stade de nos recherches, marquée par la linguistique de l'énonciation (Culioli, 1990 ; KerbratOrecchioni, 2002) et par la recherche de marqueurs susceptibles de rendre compte des traces linguistiques du sujet d'énonciation.

\section{Exemples d'analyses ${ }^{8}$}

Nous limitons notre illustration à des extraits de discours qui permettent de mettre l'accent, d'une part, sur la (re)négociation des représentations dans l'interaction, d'autre part, sur la manière dont l'énonciateur peut être amené à moduler ses relations aux représentations instituées et de situer celles-ci "entre l'adhésion absolue et la simple référence" (Py, 2000 : 119). Les deux aspects retenus permettent de mettre à jour de nouvelles formes de variabilité des RS qui contribuent à affiner la description issue de l'étude des RS stabilisées.

Dans ce passage, extrait des enquêtes dans une filiale française d'une firme allemande, l'énonciateur est amené à s'exprimer sur les pratiques linguistiques en interne.

\section{Séquence 9 : Chef des ventes, dans une filiale française d'un groupe allemand}

\begin{abstract}
KZ : au sein de l'entreprise * vous parlez le dialecte/ WUB : ici au niveau du siège on parle français * à moins qu'on convoque des vendeurs alsaciens enfin des vendeurs de l'ancienne génération on va dire * qui parlent un peu l'alsacien auquel cas on parle * on est amené à parler en alsacienl
\end{abstract}

L'énonciateur a d'abord recours à une référence commune, immédiatement disponible, qui met en avant l'usage du français dans une filiale d'un groupe allemand. Elle s'exprime dans l'assertion: "ici, au niveau du siège, on parle français". En raison de son expérience

\footnotetext{
${ }^{8}$ Ces exemples sont extraits des recherches menées avec Tsamadou-Jacoberger dans le cadre du projet Dylan.
} 
personnelle, il est toutefois amené à reformuler sa position et à recontextualiser l'objet de discours. Le passage à une autre assertion : "auquel cas on est amené à parler l'alsacien", qui est contradictoire avec la première, vient restreindre la validité de la prédominance de l'usage $\mathrm{du}$ français en même temps qu'elle remet en question la stabilité des représentations partagées.

Pour illustrer une autre forme de dynamique ou de variation, nous avons retenu des séquences (10-14) où les énonciateurs sont amenés à moduler leurs relations aux représentations majorantes de l'anglais qui constituent l'un des traits saillants de leur groupe. Les quatre exemples retenus tendent à montrer comment le discours fournit aux énonciateurs la possibilité de prendre ou non leur distance par rapport aux disponibilités sociales de leur groupe.

La séquence qui suit illustre la position d'un décideur qui reprend à son compte la représentation qui circule (et qu'il contribue à faire circuler) dans son groupe d'appartenance :

\section{Séquence 10 : PDG, PME française}

$A B$ : est-ce que * du point de vue des connaissances et des pratiques * y a des langues qui sont plus importantes que d'autres/

SO1: de façon générale dans l'économie mondiale/ c'est l'anglaisl je pense que c'est l'anglais et ça restera l'anglaisl

Dans ce fragment, on se situe dans la modalité de l'assertion et dans la modalité appréciative. Le contenu propositionnel ("l'anglais est la langue la plus importante") est tenu pour vrai. Sa prise en charge par l'énonciateur est soulignée par l'ajout de l'atténuateur "je pense" et par la reprise de "c'est l'anglais". L'emploi du verbe "rester" au futur ne fait qu'accentuer la certitude de l'énonciateur par rapport à la validation positive du contenu propositionnel, ce qui témoigne de la prise en charge sans réserve de la référence commune.

La même pré-construction, prête à l'emploi, peut susciter des prises de position plus réservées qui prennent le plus souvent la forme d'une argumentation (cf. séquences 11 et 12). L'argument retenu est, toutefois, de nature diverse.

\section{Séquence 11 : Chef de région des vendeurs, filiale française d'un groupe allemand}

$K Z$ : est-ce qu'il y a une langue qu'il serait indispensable de savoir quelle que soit l'entreprise * quel que soit le secteur/

WUB : indispensable/ l'anglais ** je pense ** puisque c'est une langue internationalel 
Dans cette séquence, l'énonciateur fait d'abord appel à la représentation partagée par / dans son groupe dont il ne peut / veut pas se dissocier. Avec "je pense", il évalue les chances de validation de la relation prédicative : "l'anglais est une langue indispensable". Manifestant une forme d'incertitude, voire d'insécurité, il introduit alors une proposition qui justifie l'énonciation de la proposition précédente. Pour ce faire, il ne peut avoir recours qu'à une formule préconstruite (stéréotypée) qui est diffusée et partagée dans son groupe et qu'il ne remet pas réellement en question.

Dans le prochain exemple, l'argument invoqué relève d'une décision extérieure au sujet.

\section{Séquence 12 : Chef de vente régionale, filiale française du groupe allemand.}

$K Z$ : est-ce que vous avez l'impression qu'il y a des langues qui ont pris plus d'importance que d'autres dans votre entreprise/

WUH : ouais *** parce qu'on a décrété * quoi/ ** l'anglais par exemple mais parce que c'est justement * on a décrété que c'était une langue internationale d'échanges * en termes commercial * c'est la langue commercialel

Ici, la réponse de l'énonciateur concernant le statut de l'anglais est fondée non pas sur sa propre évaluation, mais sur l'évaluation générale formulée par "on a décrété". L’emploi de "on" (qui renvoie à un groupe dont il s'exclut), d'une part, le discours indirect d'autre part, marquent que l'énonciateur, sans pouvoir se désengager complètement par rapport à la validation de l'énoncé rapporté, ne s'en porte pas garant explicitement. Il est remarquable que d'autres extraits de discours du même énonciateur témoignent de son insécurité linguistique face à la prédominance réelle ou fantasmée de l'anglais.

Afin d'illustrer les possibles écarts entre les positionnements et les degrés d'adhésion variables des énonciateurs à une représentation partagée par / dans leur groupe, nous retenons un dernier exemple, révélateur d'une distanciation explicite.

\section{Séquence 13 : PDG d'une filiale française d’un groupe américain}

$A B$ : est-ce que vous pensez que: ** enfin si vous avez des enfants: ** si vous avez des enfants * est-ce que vous allez porter une attention particulière à leur formation en langue/

WRF : ouil euh: * j'ai beauCOUP insisTÉ euh: pour QUE mes enfants apprennent * parlent correctement l'anglais $\backslash$ bon il se trouve que il se trouve que l'anGLAIS c'est actuellement la langue la plus parlée 1 et qui euh * probablement malHEUreusement euh: mange les AUtres। 
Dans cette séquence, l'énonciateur hésite ; il exprime des réserves sur la vérité et la pertinence de la proposition. "Bon", "il se trouve que", "probablement", "malheureusement" constituent les traces discursives de l'évaluation quantitative et qualitative de l'énonciateur quant à la validation du contenu propositionnel. Il apparaît que l'énonciateur déplore la prédominance de l'anglais, il n'y adhère pas et finit par marquer sa distance par rapport au contenu propositionnel avec l'appréciatif "malheureusement" et la métaphore de la langue glottophage.

Il ressort de cette brève analyse que les RS se modifient lorsqu'elles sont configurées par et pour le discours. Les énonciateurs disposent de ressources qui leur permettent de moduler leur relation aux représentations diffusées dans leur groupe, de les adapter à leur propre expérience et de les reconstruire en fonction des contingences du moment. En raison de l'objectif fixé qui consiste à évaluer l'apport des RS pour une description sociolinguistique - il s'agit là d'un aspect essentiel "en ce sens que les personnes affirment ainsi l'originalité de leur position tout en se rattachant à une communauté culturelle et linguistique" (Py, 2003 : 17). Or c'est bien dans la tension entre ces deux pôles que se manifestent la complexité et la diversité des phénomènes observés.

\section{Conclusion}

Si l'on retient, comme nous l'avons fait, les RS comme ressources ou comme outils de la description sociolinguistique, il convient de prendre un certain nombre de précautions théoriques et méthodologiques.

Á moins de réduire considérablement la complexité des RS et de la situation de plurilinguisme que l'on cherche à décrire, on ne peut se limiter à mettre en évidence les seuls éléments stables et partagés des RS et ce qui relève de la "monophonie collective" (Billiez / Millet, 2003 : 47). La dimension sociale des représentations n'exclut ni la variation, ni la diversité. Il convient, en conséquence, de ne pas procéder à une rupture épistémologique entre les approches psychosociologique et discursive dont la complémentarité est essentielle. Dans la démarche que nous avons adoptée, elles se présupposent et s'éclairent réciproquement : les RS partagées et plus stables permettent de mieux comprendre les fonctionnements discursifs des énonciateurs particuliers qui, à leur tour, viennent enrichir la notion même de RS (dans sa conception psychosociologique). 


\section{Références}

\section{Bibliographie}

Abric, J.-C. (1994). "Les représentations sociales : aspects théoriques". In Abric, J.-C. (dir.). Pratiques sociales et représentations. Paris : Presses Universitaires de France.

Blanchet, Ph. (2000). La linguistique de terrain. Méthode et théorie. Une approche ethnosociolinguistique. Rennes : Presses Universitaires de Rennes.

Blanchet, Ph. (2005). «Minoration, minorisation, minorités: Essai de théorisation d'un processus complexe », in Huck, D., Blanchet, Ph., Minoration, minorisation, minorités. Études exploratoires. Rennes : Presses Universitaires, pp. 17-47.

Blanchet, Ph., De Robillard, D. (dir.) (2003). Langues, contacts, complexité. Perspectives théoriques et sociolinguistiques. Rennes : Presses Universitaires de Rennes.

Billiez, J., Millet, A. (2000). "Représentations sociales: trajets théoriques et méthodologiques". In Moore, D. (dir.). Les représentations des langues et de leur apprentissage. Références, modèles, données et méthodes. Paris : Didier, pp. 31-49.

Calvet, L.-J. (1999). Pour une écologie des langues du monde. Paris : Plon.

Canut, C. (2000). "Subjectivité, imaginaires et fantasmes des langues: la mise en discours épilinguistique". Langage et Société, n 93, pp. 72-97.

Canut, C., 2007. "L'épilinguistique en question". In Siouffi G., Steuckardt A. (dir.). Les linguistes et la norme. Aspects normatifs du discours linguistique. Berne : Peter Lang Verlag.

Castellotti, V., Moore, D. (2002). Représentations sociales des langues et enseignement. Strasbourg : Conseil de l'Europe.

Cavalli, M., Coletta, D., Gajo, L., Matthey, M., Serra, C. (2003). Langues, bilinguisme et représentations sociales au Val d'Aoste. Aoste : Irre.

Culiolli, A. (1990). Pour une linguistique de l'énonciation. Tomes I-III, Paris : Ophrys. 
Gajo, L. (2003). "Approche comparative des données suisses et valdôtaines". In Cavalli M., Coletta D., Gajo L., Matthey M., Serra C. (dirs). Langues, bilinguisme et représentations sociales au Val d'Aoste. Aoste : Irre.

Guimelli, Ch. (dir.) (1994). Structures et transformations des représentations sociales. Lausanne : Delachaux et Niestlé.

Huck, D., Blanchet, Ph. (dir.) (2005). Minorations, minorisations, minorités. Rennes : Presses Universitaires de Rennes.

Huck, D., Bothorel-Witz, A., Geiger-Jaillet, A. (2007). "L'Alsace et ses langues. Éléments de description d'une situation sociolinguistique en zone frontalière". In Abel, A., Stuflesser, M., Voltmer L. (dir.). Aspects of Multilingualism in Europen Border Regions : Insights and Views of Alsace, Eastern Macedonia and Thrace, the Lublin Voivodeship and South Tyrol. Bozen : Eurac.

Jodelet, D. (1989). "Représentations sociales : un domaine en expansion". In Jodelet, D. (dir.), Les représentations sociales. Paris : Presses Universitaires de France.

Juillard, C. (2007). "Le plurilinguisme, objet de la description sociolinguistique". Langage et Société, $\mathrm{n}^{\circ}$ 121-122, pp. 235-245.

Kerbrat-Orecchioni, C. (2002). L'énonciation. Paris : Armand Colin.

Le Page, R. B., Tabouret-Keller, A. (1985) Acts of Identity. Creole-based approaches to language and ethnicity. Cambridge: Cambridge University Press.

Moliner, P., Guterman, M. (2004). "Dynamique des descriptions et des explications dans une représentation sociale". Papers on Social Representations, Textes sur les représentations sociales, vol. 13, pp. 2.1-2.12. Disponible en ligne. http://www.psr.jku.at/

Moliner, P., Martos, A. (2005). "La Fonction Génératrice de Sens du Noyau des Représentations sociales : une remise en cause ?". Papers on Social Representations, Textes sur les représentations sociales, vol. 14, pp. 3.1-3.12. Disponible en ligne. http://www.psr.jku.at/

Mondada, L. (2004), "Représentations, stéréotypes, catégorisations : de nouvelles voies pour la sociolinguistique et la linguistique de l'acquisition ? ". In Gajo, L., Matthey, M., Moore, D., 
Serra, C. (dir.). Un parcours au contact des langues. Textes de Bernard Py commentés. Paris : Didier.

Moore, D. (dir.) (2003). Les représentations des langues et de leur apprentissage. Références, modèles, données et méthodes. Paris : Didier.

Py, B. (2000). «Représentations sociales et discours. Questions épistémologiques et méthodologiques », Tranel 32, pp. 5-20.

Py, B. (2003). "Introduction », in Cavalli M., Coletta D., Gajo L., Matthey M., Serra C. (dirs). Langues, bilinguisme et représentations sociales au Val d'Aoste. Aoste : IRRE-VDA, pp. 15-33.

Py, B. (2004). "Pour une approche linguistique des représentations sociales". Langages "Représentations métalinguistiques ordinaires et discours", n 154, pp. 6-19.

Singy, P. (1996). L'image du français en Suisse romande. Une enquête sociolinguistique en pays de Vaud. Paris : L'Harmattan.

\section{Annexes}

\section{Annexe 1}

Le projet "Conscience linguistique des locuteurs dialectophones alsaciens" (A. Bothorel / D. Huck), 1987 - , Département de Dialectologie alsacienne, Université Marc Bloch, Strasbourg II.

Ce projet - qui marque le début de nos recherches sur RS - a été initié par le GdR 09 du Cnrs, à la fin des années 80 , avec l'objectif de renouveler, par le biais d'une collecte ethnotextuelle, les études dialectologiques traditionnelles. En adaptant le questionnaire au terrain alsacien, nous avons focalisé l'attention sur les dialectes alsaciens en lien avec les autres variétés en présence (variétés de français, allemand). Nous cherchions, par le biais de ce que les locuteurs alsaciens pensent, disent (ou non) de leurs langues, de leurs pratiques et de celles des autres, à acquérir une vision d'ensemble du paysage sociolinguistique alsacien et des rapports des locuteurs aux langues. Cette première étude - qui a donné lieu à plus de 500 entretiens semidirectifs avec des locuteurs dialectophones aux profils et aux habitus très diversifiés - sert en quelque sorte de base de référence aux études de terrain ultérieures. Elle nous a permis de 
tracer les lignes d'une conscience ethnolinguistique collective et de mettre en relief les RS partagées des variétés en contact, des pratiques, etc.

Le questionnaire ouvert servant de trame à l'entretien comporte 7 volets : 1. la biographie sociolinguistique; 2. la délimitation de l'espace de vie et de l'espace linguistique d'appartenance ; 3. l'auto-évaluation des compétences linguistiques en alsacien, en français et en allemand; 4. les normes des trois variétés; 5. les variations (en alsacien, français, allemand) ; 6. les pratiques linguistiques et langagières ; 7. l'idéologie linguistique (rapports langue / identité, dialecte / école ...).

L'ensemble de ces données retranscrites est en cours de numérisation, afin d'être mis à disposition des chercheurs.

\section{Annexe 2}

Programme Interreg III C, Change on Borders (A.Bothorel, D. Huck, F. Bogatto, E. Mercier, A. Geiger-Jaillet), 2004-2007.

L'étude sur les RS des élèves visait plus particulièrement à cerner la motivation de l'apprentissage scolaire des langues vivantes dans des zones frontalières en Europe. En portant notre choix sur des élèves de 3ème de la filière bilangue (allemand / anglais à volume horaire égal depuis la 6ème) dans six collèges alsaciens, nous avons recherché le possible impact que les représentations peuvent avoir sur les attitudes (positives ou négatives) face aux langues et, finalement, sur l'intérêt ou la motivation qu'elles suscitent chez les apprenants. Afin d'insérer cette étude dans un cadre plus global et de ne pas dissocier le terrain scolaire de l'environnement dans lequel il est ancré, les entretiens thématiques ont non seulement porté sur les représentations des LV (allemand, anglais) et des compétences plurilingues dans le contexte d'apprentissage, mais aussi sur la constellation linguistique alsacienne (l'enquête porte pour l'essentiel sur des élèves d'origine alsacienne qui, pour autant, ne disposent pas nécessairement d'une dialectophonie active ou passive).

\section{Annexe 3}

Projet intégré Dylan, $6^{\text {ème }}$ programme-cadre (A. Bothorel, Th. Choremi, C. Truchot, I. Tsamadou-Jacoberger, D. Huck, C. Springer, doctorants) - "La gestion du plurilinguisme dans les entreprises à vocation internationale implantées en Alsace". 
Les premières recherches relevant de ce terrain, encore peu exploré, se sont inscrites dans le cadre d'un projet commun avec Rennes II sur les cultures régionales et le développement économique. Elles ont trouvé un prolongement dans le projet intégré Dylan qui porte sur la gestion de la diversité linguistique dans les institutions (scolaires, européennes) et les entreprises. La recherche de l'équipe strasbourgeoise se concentre, à partir du "discours sur" et les RS, sur l'analyse d'un double aspect : les compétences et pratiques associées au choix de langues d'une part, les "politiques" et stratégies qui les sous-tendent, d'autre part. Á ce stade du projet, nous disposons d'une douzaine d'entretiens thématiques avec des cadres supérieurs ou moyens (d'origine alsacienne) dans trois entreprises :

- une PME française en voie d'internationalisation qui diffuse des produits étrangers en France (domaine de la confiserie)

- une filiale française d'un groupe allemand et une filiale d'un groupe américain, tous deux leaders, sur le marché mondial.

\section{À propos de l'auteure}

Arlette Bothorel-Witz est professeure de dialectologie à l'Université Marc Bloch, Strasbourg 2, France et responsable du GEPE (Groupe d'Etude sur le Plurilinguisme Européen), EA 1339.

Courriel : bothorel@umb.u-strasbg.fr.

Toile : http://www.gepe-strasbourg.fr.

Adresse : Département de Dialectologie alsacienne, Université Marc Bloch, 22 rue Descartes, 67084 Strasbourg Cedex. 